\title{
Long Baseline Nulling Interferometry with the Keck Telescopes: a Progress Report
}

\author{
B. Mennesson ${ }^{1}$, R. Akeson ${ }^{2}$, E. Appleby ${ }^{4}$, J. Bell ${ }^{4}$, A. Booth ${ }^{1}$,
} M.M. Colavita ${ }^{1}$, S. Crawford ${ }^{1}$, M.J. Creech-Eakman ${ }^{3}$, W. Dahl ${ }^{4}$, J. Fanson $^{1}$, C. Felizardo ${ }^{2}$, J. Garcia ${ }^{1}$, J. Gathright ${ }^{4}$, J. Herstein ${ }^{2}$, E. Hovland ${ }^{1}$, M. Hrynevych ${ }^{4}$, E. Johansson ${ }^{4}$, C. Koresko ${ }^{2}$, D. Le Mignant $^{4}$, R. Ligon ${ }^{1}$, R. Millan-Gabet ${ }^{2}$, J. Moore ${ }^{1}$, C. Neyman ${ }^{4}$, D. Palmer ${ }^{1}$, T. Panteleeva ${ }^{4}$, C. Paine ${ }^{1}$, S. Ragland ${ }^{4}$, L. Reder $^{1}$, A. Rudeen ${ }^{4}$, T. Saloga ${ }^{4}$, E. Serabyn ${ }^{1}$, M. Shao ${ }^{1}$, R. Smythe ${ }^{1}$, K. Summers ${ }^{4}$, M. Swain ${ }^{1}$, K. Tsubota ${ }^{4}$, C. Tyau ${ }^{4}$, G. Vasisht ${ }^{1}$, P. Wizinowich ${ }^{4}$, and J. Woillez ${ }^{4}$

${ }^{1}$ Jet Propulsion Laboratory, California Institute of Technology, 4800 Oak Grove Drive, Pasadena CA 91109-8099

${ }^{2}$ Michelson Science Center, California Institute of Technology, 770 South Wilson Avenue, Pasadena CA 91125

${ }^{3}$ New Mexico Tech, Dept of Physics, 801 Leroy Place, Socorro, NM 87801

${ }^{4}$ W.M. Keck Observatory, California Association for Research in Astronomy, 65-1120 Mamalahoa Highway, Kamuela, HI 96743

\begin{abstract}
The Keck Interferometer Nuller (KIN) is one of the major scientific and technical precursors to the Terrestrial Planet Finder Interferometer (TPF-I) mission. KIN's primary objective is to measure the level of exo-zodiacal mid-infrared emission around nearby main sequence stars, which requires deep broad-band nulling of astronomical sources of a few Janskys at 10 microns. A number of new capabilitites are needed in order to reach that goal with the Keck telescopes: mid-infrared coherent recombination, interferometric operation in "split pupil" mode, N-band optical path stabilization using K-band fringe tracking and internal metrology, and eventually, active atmospheric dispersion correction. We report here on the progress made implementing these new functionalities, and discuss the initial levels of extinction achieved on the sky.
\end{abstract}

Keywords. instrumentation: interferometers, infrared: stars, stars: circumstellar matter.

\section{Introduction}

The main science objective of KIN is to measure the level of exo-zodiacal dust emission around nearby main sequence stars in the mid-infrared (Serabyn, Colavita \& Beichman 2000), a key design parameter for the Darwin (Léger et al. 1996) and TPF-I (Beichman et al. 1999) space missions. The basic requirement for KIN is to be able to detect exozodiacal dust emission down to 30 solar zodi levels around a solar analog located at $10 \mathrm{pc}$. In this case, the exozodi signal is roughly $1.310^{-3}$ times fainter than the stellar one at 10 microns, and arises mostly from the inner $1 \mathrm{AU}$ region around the star. This clearly indicates that the null depth requirement with KIN is of the order of $10^{-3}$, or that the null measurements need to be calibrated down to this level. Unlike other midinfrared facilities, such as SPITZER for instance, KIN's spatial scales are specifically adapted to the detection of the very inner material around nearby stars. KIN's outer working angle is defined by the smallest of three fields: the unvignetted field of view 


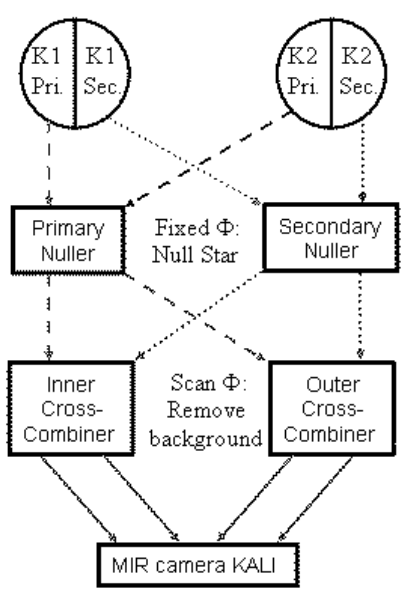

Figure 1. Conceptual KIN measurement architecture (see text).

of the interferometer, the coherent field of view fixed by KIN's spectral resolution, and the final science camera field stop. The latter is the smallest, corresponding to a field of $\simeq 500$ mas around the science target. This allows observation of the central $2.5 \mathrm{AU}$ of a dust disk around a star at $10 \mathrm{pc}$, precisely the region of interest for exo-zodiacal dust characterization. The spatial resolution of KIN is given by the first constructive peak of the sky null transmission pattern, which is $\lambda / 2 B=12$ mas, when observing at $\lambda=10$ microns, with the Keck baseline $\mathrm{B}=85 \mathrm{~m}$. This corresponds to $0.12 \mathrm{AU}$ at $10 \mathrm{pc}$, meaning that the inner brighter region of the disk is accessible for all targets of the TPF sample.

In addition to strong stellar light rejection, very precise background estimates are necessary in order to measure accurate null levels from the ground at 10 microns. This calls for a 2-step removal process: starlight suppression and then background subtraction, as illustrated in figure 1. Each of the Keck telescope pupils is divided into 2 sub-apertures, defining primary and secondary beams. The primary beams of each telescope are nulled together, as well as on the secondary side. The residual primary and secondary nulled beams are then coherently mixed together with a modulated phase shift, using the "cross combiner" beam splitters. As a result, the residual light coming from the astronomical source becomes an "AC" signal, easily separated from the incoherent background signal which remains a "DC" signal. The final null measurement is still affected by the background noise, and its fluctuations at the modulation frequency.

It is worth noting that this scheme is fairly similar to the phase chopping technique Woolf and Angel 1997, Mennesson, Léger \& Ollivier 2005) considered for the TPF-I and Darwin missions.

\section{From the laboratory to the sky}

The 4 beam nulling/phase modulation scheme described above was tested and implemented gradually over the past 2 years. 


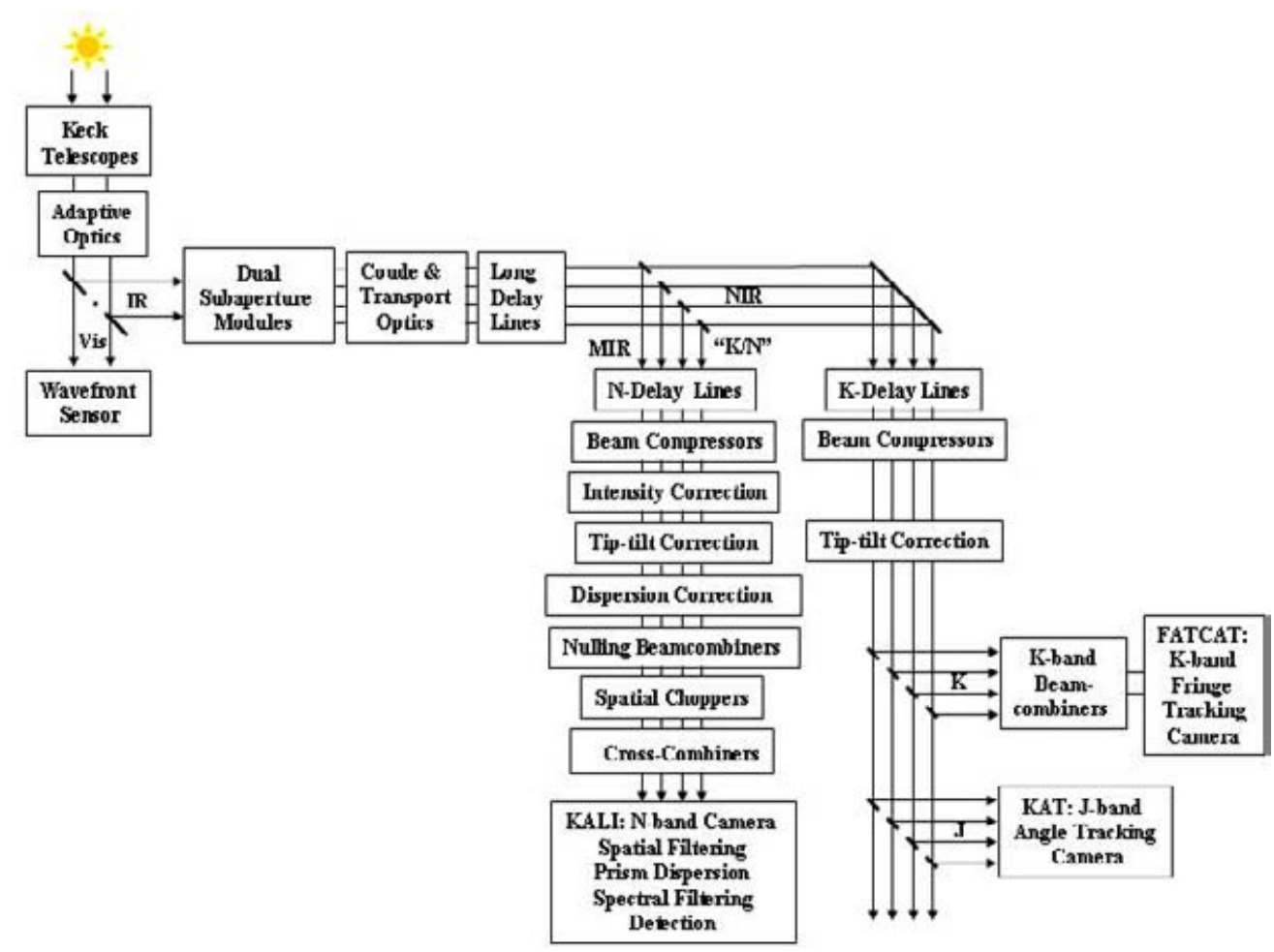

Figure 2. System level block diagram of the KIN, and the starlight path through the system. Not shown explicitely are the MIR nuller (8) and cross-combiner (2) beam splitters, which lie in the boxes labeled "Nulling Beam Combiners" and "Crosscombiners".

A basic physics experiment was set-up at JPL, using a pair of inverted beam splitters for symmetric beam combination (Serabyn \& Colavita 2001) and dielectric phase plates for achromatic phase shifting. Deep thermal infrared wide band nulls were obtained in 2003 (Mennesson et al. 2003), basically validating the optics approach. A more realistic set-up was then integrated and tested in the laboratory, with long airpaths above various optical tables. It included most of the components needed for nulling at the Keck observatory: 4-N band delay lines, $4 \mathrm{~K}$-band delay lines, a 4 beam nulling bread-board, laser metrology, and a helium cooled science camera (Creech-Eakman et al. 2003). In May 2004, wideband $(8-10 \mu \mathrm{m})$ deep nulls $\left(<10^{-3}\right)$ were obtained and stabilized over 5 minutes, with repeatability maintained over 48 hours (Serabyn et al. 2004).

At this point, the nulling components were shipped to Hawaii for integration with the Keck Interferometer optical train, which is summarized in figure 2. The initial phase of the install was completed in August 2004, culminating with the first thermal infrared fringes between the Keck telescopes. These observations were carried out in regular visibility mode. They confirmed the instrument's ability to derive dust shell properties (optical depth profile, spatial extent) in the favorable case of stars with known large infrared excesses, such as the dusty AGB star RS CrB (Mennesson et al. 2005).

Subsequent developments targeted nulling more specifically. A split pupil module was installed at the telescope level, in order to define the primary and secondary beams issued from each Keck telescope. Simultaneous fringes between primary beams and secondary beams were obtained in October 2004, as well as cross fringes between beams coming from a given telescope. Further details on the metrology and real time control systems 


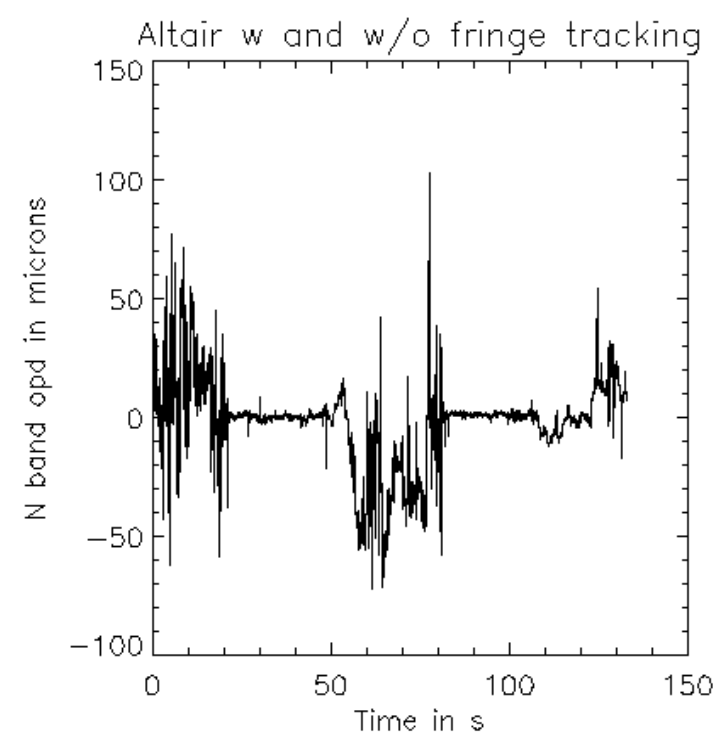

Figure 3. Optical path difference (opd) measured around 10 microns. The "quite" periods with small opd fluctuations correspond to the time intervals when the K-band phase is "fed forward" to the N-band delay line. No LASER metrology used during the sequence.

installed on the KIN can be found in Serabyn et al. 2005. A key functionnality for deep nulling is the K-band fringe tracking feed forward capability. KIN's target stars are too faint at 10 microns to allow fast fringe tracking in the N-band. The fast opd stabilization is instead carried out at 2 microns, - where stellar photons are plentiful. The phase information is then "fed forward" to the N-band delay lines, using LASER metrology to monitor the non-common $\mathrm{K}$ vs $\mathrm{N}$ band airpath fluctuations. The dramatic effect of enabling K-band feed forward is illustrated in figure 3. Only feed forward allows opd stabilization at the level required for deep nulling.

\section{Current performance and future improvements}

The current level of broad-band extinction achieved with KIN (Serabyn et al. 2005) is illustrated in figure 4. It shows the nulls measured on Vega, a bright A0V star (37 Jsky at 10 microns), as a function of wavelength, and for each of the two KALI ports presently used (open and filled squares). The dotted line shows for comparison the geometric null depth expected as a function of wavelength, representing Vega as a 3.28 mas uniform disk using the limb darkened diameter derived by Ciardi et al. (2001). It is unclear at this point whether the extra leakage detected with KIN is purely instrumental or of astrophysical origin. Yet, assuming that all of the extra leakage is of intrumental origin, figure 4 shows that a broadband instrumental null depth of 0.01 or better has been achieved between 8 and 10 microns.

Although accessible via accurate calibration, the detection of structures fainter than the geometric stellar leakage - fixed by the finite stellar disk size- is of course difficult. Faint structures will be more readily detectable around stars small enough to provide low geometric nulls in the first place. Achievable null depth and sensitivity are then closely related, as shown in figure 5. Considering naked stars as uniform spherical blackbodies, hotter stars are also less resolved for a given flux, making early type stars more favorable targets. Over the last year, KIN targets have evolved from bright red giants - with strong 


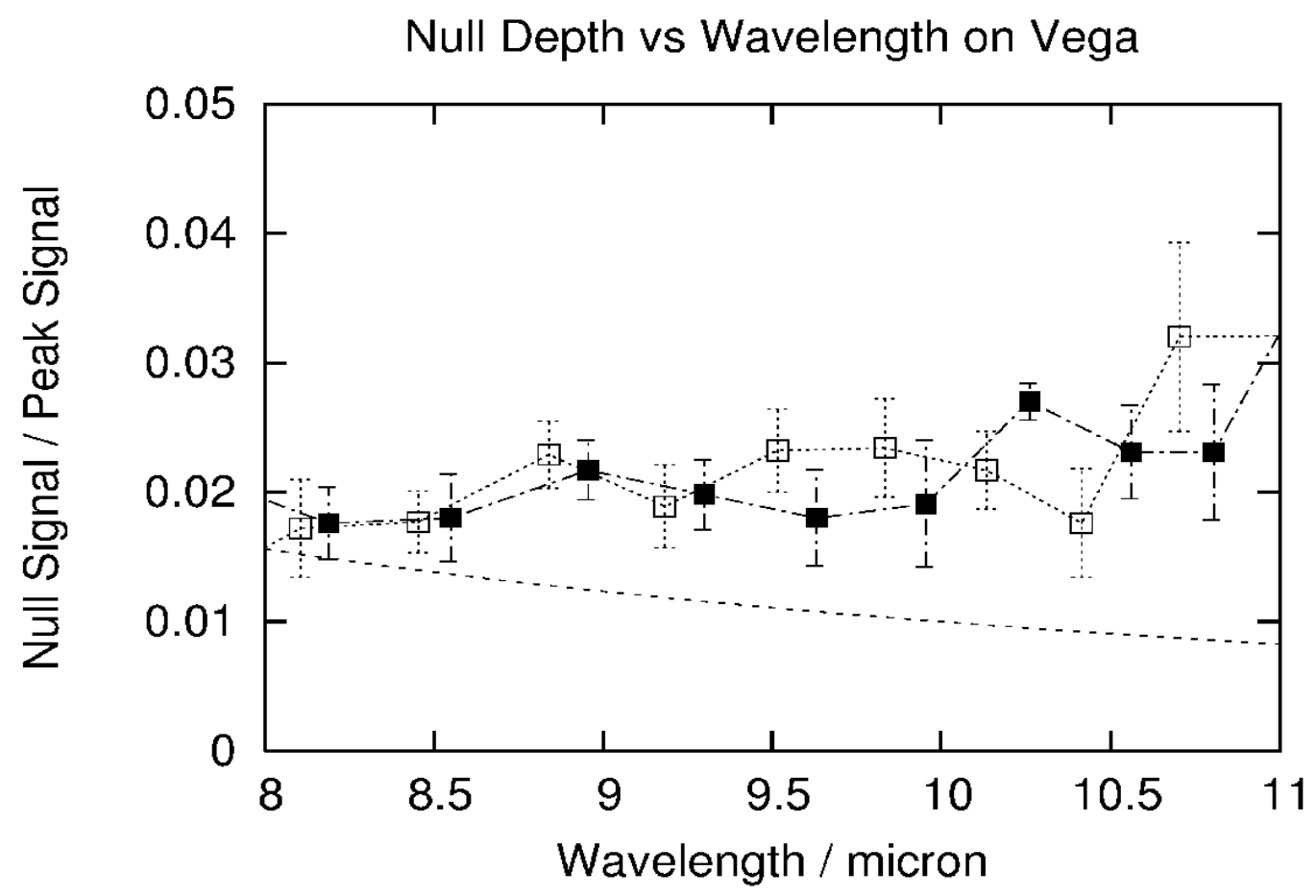

Figure 4. Raw null measurements obtained on Vega.

and easily detectable infrared excesses - to main sequence stars in the 15-30 Jy range. Clearly the main objective of future KIN developments will be to reach the top of the TPF sample region, with stellar fluxes clustered around a few Jy (lower light region of figure 5).

\section{Summary}

All of the equipment is now in place for deep thermal infrared nulling with the Keck Interferometer. Broadband instrumental nulls of at least 100:1 have already been demonstrated on bright targets of a few tens of Jy.Future efforts will concentrate on achieving similar levels of instrumental nulls on fainter stars. The final goal is to reach a sensitivity of a few Jy, as needed to survey the TPF targets core.

\section{Acknowledgements}

The Keck Interferometer is funded by the National Aeronautics and Space Administration (NASA). Part of this work was performed at the Jet Propulsion Laboratory, California Institute of Technology, and at the Michelson Science Center (MSC), under contract with NASA.

\section{References}

Beichman, C.A., Woolf, N.J., \& Lindensmith, C.A. 1999, The Terrestrial Planet Finder: a NASA Origins Program to Search for Habitable Planets, JPL Publication 99-3

Ciardi, D.R. et al. 2001, ApJ, 559, 1147 


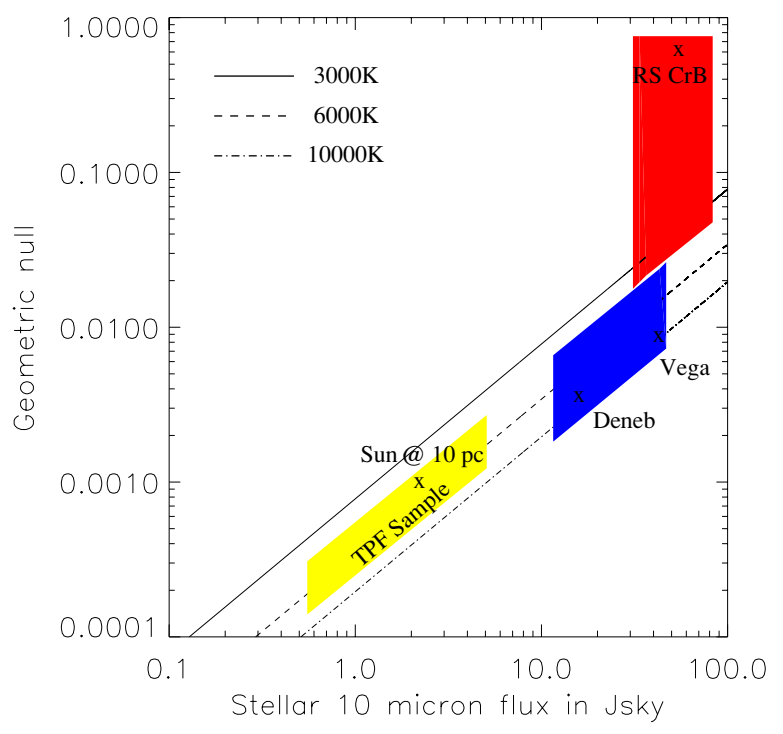

Figure 5. Null as a function of 10 micron flux for a naked star considered as a blackbody at various temperatures.

Creech-Eakman, M.J. et al. 2003, SPIE Conf. Series 4841, 330

Léger, A., Mariotti, J.M., Mennesson, B., Ollivier, M. et al. 1996, Icarus, 123, 249-255

Mennesson, B. et al. 2003, in Conf. Towards Other Earths: DARWIN/TPF and the Search for Extrasolar Terrestrial Planets, ed. M. Fridlund \& T. Henning, (Noordwijk: ESA-SP-539), 525

Mennesson, B., Léger, A., \& Ollivier, M. 2005, Icarus, 178, 2, 570

Mennesson, B. et al. 2005, ApJL in press

Serabyn, E., Colavita M. M., \& Beichman C.A. 2000, ASP Conf. Series 196, 357, eds M.L. Sitho, A.L. Sprague \& D.K. Lynch

Serabyn, E. \& Colavita, M. M. 2001, Applied Optics, 40, 1668

Serabyn, E. et al. 2004, SPIE Conf. Ser. 5491, 806, ed. W.A. Traub

Serabyn, E. et al. 2005, SPIE Conf Series 5905, in press.

Woolf, N.J. \& Angel, J.R.P. 1997, ASP Conf. Series 119, 285-292, D. R. Soderblom, ed.

\section{Discussion}

YvES RABBIA: What are the next developments expected with KIN?

B. Mennesson: We will concentrate on improving the sensitivity. We plan to do this by lowering our control loop bandwidth on the 10 micron phase. In order to keep the same nulling level, we will need to reduce the $\mathrm{K}$ versus $\mathrm{N}$ band phase drifts due to atmospheric dispersion and residual differential paths unseen by the present LASER metrology.

AlAIN LÉGER: How does your current performance translate in terms of exo-zodi detection capability?

B. Mennesson: In the case of a solar system twin located at $10 \mathrm{pc}$, only $0.004 \%$ of the total 10 micron flux $(\simeq 2 \mathrm{Jy})$ comes from the exo-zodi. A calibrated null of 100:1 then corresponds to a level of 250 zodis around a solar type star. 